\title{
Effects of Poynting-Robertson Drag and Oblateness on the Stability of Photogravitational Restricted Three-Body Problem
}

\author{
Jaiyeola Sefinat. B. ${ }^{1^{*}}$, Abdulrazaq Abdulraheem ${ }^{2}$ and Titiloye, Emmanuel O. ${ }^{3}$ \\ ${ }^{1}$ Department of Physical Sciences, College of Natural Sciences, Al-Hikmah University, P.M.B 1601,Ilorin, Kwara \\ State, Nigeria \\ ${ }^{2}$ Department of Statistics and Mathematical Sciences,Kwara State University, Malete, Kwara State, Nigeria. \\ ${ }^{3}$ Department of Mathematics, University, of Ilorin, PMB 1515, Ilorin, Kwara State, Nigeria. \\ Email: sefinajaiyeola15@gmail.com
}

\begin{abstract}
This research work investigates the effect of Poynting-Robertson (P-R) drag on the stability of the triangular libration points of the Restricted Three Body Problem (RTBP) when the primaries are considered to be oblate spheroid as well as sources of radiation. We established the equations of motion, which incorporated the perturbing forces, for the system. Using linear approximations, we obtained the triangular libration points in terms of the perturbing parameters and found that they were affected by them. Due to the nature of the characteristics equation corresponding to the variational equations of motion of the system obtained, we employed Murray's criteria and concluded that the triangular libration points remained unstable.
\end{abstract}

Keywords: Stability, libration points, oblateness, P-R drag, RTBP.

\section{Introduction}

The Restricted Three Body Problem (RTBP) is the motion of an infinitesimal body in the field of two bodies of finite masses which revolve in circles around their barycenter. In the classical case, [37] showed that the RTBP possesses five equilibrium points, three collinear $\left(L_{1}, L_{2}, L_{3}\right)$ which are seen to be unstable and two triangular $\left(L_{4}, L_{5}\right)$ points which are stable for $0 \leq \mu \leq \mu_{c}$, where $\mu$ is the mass ratio and $\mu_{c}$ is the critical mass value. [33] obtained the complete solution of the problem and established the existence and stability of equilibrium points for all values of mass ratio. [28] also analysed the stability of the RTBP. They formulated the equation of motion using the Lagrange's-Hamiltonian technique. Here, perturbing factors (radiation, varying masses, atmospheric drag, solar wind drag, oblateness, triaxiality, coriolis and centrifugal forces, etc.) were not put into consideration in determining the stability of the RTBP.

One of the immediate generalizations of the RTBP is the photogravitational problem. Several authors have generalized the classical case by taking into account the photogravitational effect on the RTBP. [24] was the first to formulate the photogravitational RTBP. He studied the linear stability of the five libration points in the planar case. [15] studied the stability in the Liapunov sense with one of the primaries radiating. [21] showed the astronomical applications of the stability problem by considering the influence of radiation pressure from the sun in the planet-satellite-particle system. [14], [10], [12], [16] and [17] also worked on the similar problem.

These results were further modified by considering the shape of the planetary bodies. [20], [39], [30], [13], [1], [4] and [35] studied the stability of the RTBP with either one or both of the primaries being oblate spheroid and radiating.

[29], [6], [31], [38], [32], [2] and many others investigated the case of triaxial primaries with radiating effect.

In addition, the effect of small perturbations in the coriolis and centrifugal forces on the motion around the RTBP was considered. [38], [37], [5], [3] found that the stability behaviour of collinear points does not change despite the introduction of small perturbation in the coriolis and centrifugal forces, 
oblateness and radiation pressure parameters while the triangular points are stable for $0 \leq \mu<\mu_{c}$ and unstable for $\mu_{c} \leq \mu \leq \frac{1}{2}$. [36] studied the combined effect of perturbation, radiation and oblateness on the nonlinear stability of RTBP.

The importance of radiation influence on the planetary and binary systems caught the attention of many researchers. [23], was the first to describe the effect of radiation pressure force when he considered the effect of absorption and subsequent re-emission of sunlight by small isolated particles in the solar system. His work was later modified by [26], when he explained the effect of total radiation force on a particle due to radiation source. He used a precise relativistic treatment of the first order in the ratio of the velocity of the particle to that of light $\frac{\bar{v}}{c}$ and gave the expression for the net drag force which opposes the direction of motion as

$$
F=F_{p}\left\{\frac{\vec{v}}{r}-\frac{\vec{v} \vec{r} \vec{r}}{c r r}-\frac{\vec{r}}{c}\right\}
$$

where $F_{p}=\frac{3 L M}{16 \pi R^{2} \rho A C}$ denotes the measure of radiation pressure and

$\vec{r}$ is the position vector of $p$ with respect to the sun $S$

$\vec{v}$ is the corresponding velocity

$c$ is the velocity of light

$L$ is the luminosity of the radiating body

$M$ is the mass of the particle

$\rho$ is the density of the particle

$A$ is the cross section of the particle.

The first term in (1) expresses the radiation effect and the last two terms constitute the P-R drag effect.

[7] and [27] studied the existence and stability of equilibrium positions by taking into account the P$\mathrm{R}$ drag force with only one of the primaries radiating. [7] established that six libration points exist at most, five of which lie on the orbit plane while the sixth point falls out of the plane of motion and is perpendicular to the orbital plane. They concluded that the P-R effect rendered unstable points that were found to be conditionally stable in the classical case. [25] investigated numerically the same problem taking both primaries to be radiating. They found that the collinear points deviated from the axis while the triangular points were no longer symmetrical. [22] and [19] discussed the effect of solar wind drag on the stability of RTBP. [8] studied the out of plane equilibrium points of a passive micro size particle and their stability in the field of radiating binary stellar system. [11] perfomed an analysis of the circular RTBP under the effect of the dissipative force, P-R drag. He did not consider the second component of this drag force i.e. doppler shift of solar radiation and discovered that the stationary solutions were unstable for $\mu<\mu_{c}$.

[9] generalized the RTBP by considering the bigger primary to be an oblate spheroid and the smaller one radiating with $\mathrm{P}-\mathrm{R}$ effect. On the other hand [34] investigated the effect of this drag when the smaller primary is an oblate spheroid and the bigger one a source of radiation with P-R drag effect. They found that due to the presence of P-R drag the triangular points were unstable.

Due to their remarkable and dynamical results on the effects of P-R drag forces on the motion around the orbit of the satellite (natural/artificial) and its significance in dealing with problems in astrophysics, we hereby generalize their work further and propose a new model to study the effect of P-R drag on the stability of the triangular libration points in the RTBP when the primaries are oblate spheroid as well as sources of radiation.

The paper is organized as follows: section 2 presents the equations of motion of our model, section 3 deals with the location of libration points. In section 4, we examine the stability character of the problem using Murray's idea and section 5 entails the discussion and conclusion. 


\section{Equations of Motion}

With reference to an inertia or fixed coordinates XYZ, let $m_{1}$ and $m_{2}$ be the masses of the primaries $S_{1}, S_{2}$ respectively and $\mathrm{M}$ be the mass of the infinitesimal mass $P$. We introduce a rotating coordinate system Oxyz with the origin $\mathrm{O}$ at the barycenter of the primaries in which the axes rotate relative to the inertia space with an angular velocity $\omega=n \hat{k}$.

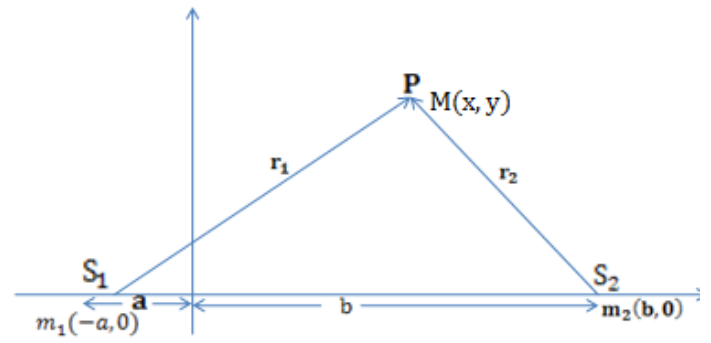

Figure 1. Coordinates of the primaries w.r.t. the inertia frame of reference OXY

We adopt the notation and terminology of [37] in which the distance between the primaries is taken to be equal to one; the sum of the masses of the primaries is also taken as 1 so that if $m_{2}=\mu$, then $m_{1}=1-\mu$ where $\mu$ is the mass ratio parameter and $\mu=\frac{m_{2}}{m_{1}+m_{2}}$. The unit of time is also chosen so as to make the gravitational constant $\mathrm{G}$, to be equal to one. The oblateness coefficient is $A_{1}=\frac{a e_{1}^{2}-a p_{1}^{2}}{5 R^{2}}, A_{2}=\frac{a e_{2}^{2}-a p_{2}^{2}}{5 R^{2}}[20]$, where $0<A_{i} \ll 1, a e_{i}, a p_{i}, i=1,2$ are the equatorial and polar radii respectively and $R$ is the distance between the primaries. The velocity of light $c$ is given as $c=c_{d}$ and the mass reduction factor expressed in terms of particle radius $a$, density $\delta$ and its solar radiating pressure efficiency factor $\kappa$ (in chs unit) are taken to be constants so that the mass reduction factor $\quad q=1-\frac{5.6 \times 10^{-5}}{a \delta} \kappa, \quad$ and $0<\left(1-q_{i}\right) \ll 1, \quad i=1,2$ for the bigger and smaller primary respectively.

In the dimensionless synodic coordinate system the equations of motion of the infinitesimal mass in the $x-y$ orbital plane $(\mathrm{z}=0)$ is

$$
\begin{aligned}
& \ddot{x}-2 n \dot{y}=\Omega_{x} \\
& \ddot{y}-2 n \dot{x}=\Omega_{y}
\end{aligned}
$$

where

$$
\begin{aligned}
\Omega_{x}= & n^{2} x-\frac{(1-\mu)(x+\mu) q_{1}}{r_{1}^{3}}-\mu \frac{(x+\mu-1) q_{2}}{r_{2}^{3}}-\frac{3(1-\mu)(x+\mu) A_{1}}{2 r_{1}^{5}}-\frac{3 \mu(x+\mu-1) A_{2}}{2 r_{2}^{5}} \\
& \left.\left.-\frac{W_{1}}{r_{1}^{2}}\left\{\frac{x+\mu}{r_{1}^{2}}[\dot{x}(x+\mu)+y \dot{y}]+\dot{x}-n y\right]\right\}-\frac{W_{2}}{r_{2}^{2}}\left\{\frac{x+\mu-1}{r_{2}^{2}}[\dot{x}(x+\mu-1)+y \dot{y}]+\dot{x}-n y\right]\right\} \\
\Omega_{y}= & n^{2} y-\frac{(1-\mu) q_{1} y}{r_{1}^{3}}-\mu \frac{q_{2}}{r_{2}^{3}}-\frac{3(1-\mu) y A_{1}}{2 r_{1}^{5}}-\frac{3 \mu y A_{2}}{2 r_{2}^{5}}-\frac{W_{1}}{r_{1}^{2}}\left\{\frac{y}{r_{1}^{2}}[\dot{x}(x+\mu)+y \dot{y}]+[\dot{y}+n(x+\mu)]\right\} \\
& -\frac{W_{2}}{r_{2}^{2}}\left\{\frac{y}{r_{2}^{2}}[\dot{x}(x+\mu-1)+y \dot{y}]+[\dot{y}+n(x+\mu-1)]\right\}
\end{aligned}
$$




$$
\begin{aligned}
& r_{1}^{2}=(x+\mu)^{2}+y^{2} \\
& r_{2}^{2}=(x+\mu-1)^{2}+y^{2} \\
& W_{1}=\frac{(1-\mu)\left(1-q_{1}\right)}{c_{d}} \\
& W_{2}=\frac{(1-\mu)\left(1-q_{2}\right)}{c_{d}}
\end{aligned}
$$

The mean motion $n$ is given as

$$
n^{2}=1+\frac{3 A_{1}}{2}+\frac{3 A_{2}}{2}
$$

\section{$3 \quad$ Location of the Triangular Libration Points}

The libration points are the solution of the equations (2) and (3), when the velocity and acceleration are equal to zero ( $\dot{x}=\dot{y}=\ddot{x}=\ddot{y}=0)$, that is,

$$
\begin{gathered}
\Omega_{x}=n^{2} x-\frac{(1-\mu)(x+\mu) q_{1}}{r_{1}^{3}}-\mu \frac{(x+\mu-1) q_{2}}{r_{2}^{3}}-\frac{3(1-\mu)(x+\mu) A_{1}}{2 r_{1}^{5}} \\
-\frac{3 \mu(x+\mu-1) A_{2}}{2 r_{2}^{5}}+\frac{n W_{1} y}{r_{1}^{2}}+\frac{n W_{2} y}{r_{2}^{2}}=0 \\
\Omega_{y}=n^{2} y-\frac{(1-\mu) q_{1} y}{r_{1}^{3}}-\mu \frac{q_{2} y}{r_{2}^{3}}-\frac{3(1-\mu) y A_{1}}{2 r_{1}^{5}}-\frac{3 \mu y A_{2}}{2 r_{2}^{5}}-\frac{n W_{1}(x+\mu)}{r_{1}^{2}}-\frac{n W_{2}(x+\mu-1)}{r_{2}^{2}}=0
\end{gathered}
$$

The triangular libration points are the solution of equations (6) and (7) when $y \neq 0$.

In the absence of oblateness $\left(A_{1}, A_{2}=0\right)$ and the P-R drag $W_{1}, W_{2}=0$ the equations above reduce to the photogravitational RTBP so that

$$
r_{1}=q_{1}^{1 / 3} \text { and } r_{2}=q_{2}^{1 / 3}
$$

Let $\left|\varepsilon_{1}\right| \ll 1$ and $\left|\varepsilon_{2}\right| \ll 1$ be small perturbation in $r_{1}$ and $r_{2}$ respectively due to the presence of oblateness and P-R drag. Then

$$
r_{1}=q_{1}^{1 / 3}+\varepsilon_{1} \text { and } r_{2}=q_{2}^{1 / 3}+\varepsilon_{2}
$$

Furthermore, we let $q_{1}=\left(1-\beta_{1}\right), \quad q_{2}=\left(1-\beta_{2}\right), \quad\left(\left|\beta_{1}\right|,\left|\beta_{2}\right| \ll 1\right)$ so that by putting these in equation (4) and solving simultaneously for $\mathrm{x}$ and $\mathrm{y}$, considering only liner terms of small quantities, we get

$$
x=\frac{1}{2}-\mu-\frac{\beta_{1}}{3}+\frac{\beta_{2}}{3}+\varepsilon_{1}-\varepsilon_{2}
$$

or

$$
x=x_{o}\left\{1+\frac{\varepsilon_{1}-\varepsilon_{2}}{x_{o}}\right\}
$$

where

and

$$
x_{o}=\frac{1}{2}-\mu-\frac{\beta_{1}}{3}+\frac{\beta_{2}}{3}
$$

$$
y=y_{o}\left(1+\frac{\varepsilon_{1}}{2 y_{o}^{2}}+\frac{\varepsilon_{2}}{2 y_{o}^{2}}\right)
$$

where

$$
y_{o}= \pm \frac{\sqrt{3}}{2}\left(1-\frac{2 \beta_{1}}{9}-\frac{2 \beta_{2}}{9}\right)
$$


Now, multiplying eqn (6) by $y,(7)$ by $(x+\mu)$ and then $(7)$ again by $(x+\mu-1)$, we have the following equations

$$
\begin{aligned}
n^{2} x y & -\frac{(1-\mu) q_{1}(x+\mu) y}{r_{1}^{3}}-\frac{\mu q_{2}(x+\mu-1) y}{r_{2}^{3}}-\frac{3(1-\mu)(x+\mu) A_{1} y}{2 r_{1}^{5}} \\
- & \frac{3 \mu(x+\mu-1) A_{2} y}{2 r_{2}^{5}}+\frac{n W_{1} y^{2}}{r_{1}^{2}}+\frac{n W_{2} y^{2}}{r_{2}^{2}}=0 \\
n^{2}(x+\mu) y & -\frac{(1-\mu) q_{1}(x+\mu) y}{r_{1}^{3}}-\frac{\mu q_{2}(x+\mu) y}{r_{2}^{3}}-\frac{3(1-\mu)(x+\mu) A_{1} y}{2 r_{1}^{5}} \\
& -\frac{3 \mu(x+\mu) A_{2} y}{2 r_{2}^{5}}-\frac{n W_{1}(x+\mu)^{2}}{r_{1}^{2}}-\frac{n W_{2}(x+\mu)(x+\mu-1)}{r_{2}^{2}}=0 \\
n^{2}(x+\mu-1) y- & \frac{(1-\mu) q_{1}(x+\mu-1) y}{r_{1}^{3}}-\frac{\mu q_{2}(x+\mu-1) y}{r_{2}^{3}}-\frac{3(1-\mu)(x+\mu-1) A_{1} y}{2 r_{1}^{5}} \\
- & \frac{3 \mu(x+\mu-1) A_{2} y}{2 r_{2}^{5}}-\frac{n W_{1}(x+\mu)(x+\mu-1)}{r_{1}^{2}}-\frac{n W_{2}(x+\mu-1)^{2}}{r_{2}^{2}}=0
\end{aligned}
$$

Subtracting (15) and (16) from (14) simultaneously, we obtain

$$
\begin{aligned}
& \mu y\left[n^{2}-\frac{q_{2}}{r_{2}^{3}}-\frac{3 A_{2}}{2 r_{2}^{5}}\right]=n W_{1}+\frac{n W_{2}}{2}+\frac{n W_{2}}{2 r_{2}^{2}}\left(r_{1}^{2}-1\right) \\
& (1-\mu) y\left[n^{2}-\frac{q_{1}}{r_{1}^{3}}-\frac{3 A_{1}}{2 r_{1}^{5}}\right]=\frac{n W_{1}}{2}-n W_{2}-\frac{n W_{1}}{2 r_{1}^{2}}\left(r_{2}^{2}-1\right)
\end{aligned}
$$

since $x+\mu=\frac{r_{1}^{2}-r_{2}^{2}+1}{2}$ and $x+\mu-1=\frac{r_{1}^{2}-r_{2}^{2}-1}{2}$.

Using equations (5), (8), (10) and (12) in (17) and simplifying (rejecting second and higher order term of small quantities, that is $\left.\left|\beta_{1}\right|,\left|\beta_{2}\right|,\left|\varepsilon_{1}\right|,\left|\varepsilon_{2}\right|,\left|A_{1}\right|,\left|A_{2}\right|,\left|W_{1}\right|,\left|W_{1}\right| \ll 1\right)$, we get

or with equation $(9)$

$$
\varepsilon_{1}=-\frac{A_{2}}{2}-\frac{n W_{1}}{6(1-\mu) y_{o}}-\frac{n W_{2}}{3(1-\mu) y_{o}} \text { and } \varepsilon_{2}=-\frac{A_{1}}{2}+\frac{n W_{1}}{3 \mu y_{o}}+\frac{n W_{2}}{6 \mu y_{o}}
$$

$$
\begin{aligned}
& \varepsilon_{1}=-\frac{A_{2}}{2}-\frac{n W_{1}}{3(1-\mu) \sqrt{3}}-\frac{2 n W_{2}}{3(1-\mu) \sqrt{3}} \\
& \varepsilon_{2}=-\frac{A_{1}}{2}+\frac{2 n W_{1}}{3 \mu \sqrt{3}}+\frac{n W_{2}}{3 \mu \sqrt{3}}
\end{aligned}
$$

Substituting these values of $\varepsilon_{1}$ and $\varepsilon_{2}$ in (10) and (12), then simplifying, we obtain

$$
x=\frac{1}{2}-\mu-\frac{\beta_{1}}{3}+\frac{\beta_{2}}{3}+\frac{A_{1}}{2}-\frac{A_{2}}{2}-\frac{n W_{1}(2-\mu)}{3 \mu(1-\mu) \sqrt{3}}-\frac{n W_{2}(1+\mu)}{3 \mu(1-\mu) \sqrt{3}}
$$

and

$$
y= \pm \sqrt{\frac{3}{2}}\left(1-\frac{2 \beta_{1}}{9}-\frac{2 \beta_{2}}{9}-\frac{A_{1}}{3}-\frac{A_{2}}{3}\right)+\frac{n W_{1}(2-3 \mu)}{9 \mu(1-\mu)}+\frac{n W_{2}(1-3 \mu)}{9 \mu(1-\mu)}
$$

Equation (19) and (20) are the coordinates of the triangular point of the modified system under investigation. 


\section{$4 \quad$ Stability of Libration Points}

To determine the stability of the libration points in the $x y$-plane, we let $\left(x_{0}, y_{0}\right)$ be the coordinate of the libration point and consider a small displacement $\xi, \eta \ll 1$ such that $x=x_{0}+\xi, y=y_{0}+\eta$ is a point in the neighbourhood of the libration point. Then, we obtain the variational equations of the system (3) as

$$
\begin{aligned}
& \ddot{\xi}-2 n \dot{\eta}=\Omega_{x}^{o}+\Omega_{x x}^{o} \xi+\Omega_{x y}^{o} \eta+\Omega_{x \dot{x}}^{o} \dot{\xi}+\Omega_{x \dot{y}}^{o} \dot{\eta}+O(2) \\
& \ddot{\eta}+2 n \dot{\xi}=\Omega_{y}^{o}+\Omega_{y x}^{o} \xi+\Omega_{y y}^{o} \eta+\Omega_{y \dot{x}}^{o} \dot{\xi}+\Omega_{y \dot{y}}^{o} \dot{\eta}+O(2)
\end{aligned}
$$

where $O(2)$ represents second and higher order terms in $\xi$ and $\eta$. The superscript (o) indicates that the second order partial derivatives are evaluated at the libration points.

If we let $\xi=A e^{\lambda t}$ and $\eta=B e^{\lambda t}$ be the trial solutions to the equations (21) then we get the characteristic equation corresponding to the variational equation of motion as

$$
\lambda^{4}+a \lambda^{3}+b \lambda^{2}+c \lambda+d=0
$$

where $a=-\left(\Omega_{x \dot{x}}^{o}+\Omega_{y \dot{y}}^{o}\right)$,

$$
\begin{aligned}
& b=4 n^{2}+\Omega_{x \dot{x}}^{o} \Omega_{y \dot{y}}^{o}-\Omega_{x x}^{o}-\Omega_{y y}^{o}-\left(\Omega_{x \dot{y}}^{o}\right)^{2}, \\
& c=\Omega_{x \dot{x}}^{o} \Omega_{y y}^{o}+\Omega_{x x}^{o} \Omega_{y \dot{y}}^{o}+2 n \Omega_{x y}^{o}-2 n \Omega_{y x}^{o}-\Omega_{y \dot{x}}^{o} \Omega_{x y}^{o}-\Omega_{y x}^{o} \Omega_{x \dot{y}}^{o}, \\
& d=\Omega_{x x}^{o} \Omega_{y y}^{o}-\Omega_{y x}^{o} \Omega_{x y}^{o}
\end{aligned}
$$

Differentiating (3) and evaluating all the second partial derivatives at libration points and considering only linear terms of small quantities, we get

$$
\begin{aligned}
& \Omega_{x x}^{o}=\frac{3}{4}-\left(\frac{1}{2}-\frac{3 \mu}{2}\right) \beta_{1}+\left(1-\frac{3}{2} \mu\right)+\left(\frac{27}{8}-\frac{24 \mu}{8}\right) A_{1} \\
& -\left(\frac{3}{2}+\frac{24 \mu}{3}\right) A_{2}-\frac{W_{1}\left(8-13 \mu+\mu^{2}\right)}{4 \mu(1-\mu) \sqrt{3}}-\frac{W_{2}\left(4-11 \mu-\mu^{2}\right)}{4 \mu(1-\mu) \sqrt{3}} \\
& \Omega_{x y}^{o}=\frac{\sqrt{3}}{4}\left[3-6 \mu-\frac{2}{3}(1+\mu) \beta_{1}+\frac{2}{3}(2-\mu) \beta_{2}+\frac{1}{2}(19-26 \mu) A_{1}+\frac{1}{2}(7-26 \mu) A_{2}\right] \\
& -\frac{W_{1}\left(8-31 \mu+27 \mu^{2}\right)}{12 \mu(1-\mu)}-\frac{W_{2}\left(4-23 \mu+27 \mu^{2}\right)}{12 \mu(1-\mu)}=\Omega_{y x}^{o} \\
& \Omega_{y y}^{o}=\frac{9}{4}+\frac{1}{2}(1-3 \mu) \beta_{1}-\frac{1}{2}(2-3 \mu) \beta_{2}+\frac{33}{8} A_{1}+\frac{33}{8} A_{2}+\frac{W_{1}\left(8-17 \mu+5 \mu^{2}\right)}{4 \mu(1-\mu) \sqrt{3}}+\frac{W_{2}\left(4-7 \mu-5 \mu^{2}\right)}{4 \mu(1-\mu) \sqrt{3}} \\
& \begin{cases}\Omega_{x \dot{x}}^{o}= & -\frac{5}{4} W_{1}-\frac{5}{4} W_{2} \\
\Omega_{x \dot{y}}^{o}= & -\frac{\sqrt{3}}{4} W_{1}-\frac{\sqrt{3}}{4} W_{2}=\Omega_{y \dot{x}}^{o} \\
\Omega_{y \dot{y}}^{o}= & -\frac{7}{4} W_{1}-\frac{7}{4} W_{2}\end{cases}
\end{aligned}
$$

Substituting these values from (23) - (26) in (22) considering only linear terms of small quantities, we obtain

$$
\begin{gathered}
a=3 W_{1}+3 W_{2} \\
b=b_{o}+b_{1}, \text { where } b_{0}=1, b_{1}=-\left(\frac{3}{2}-3 \mu\right) A_{1}+\left(\frac{3}{2}-3 \mu\right) A_{2}+\frac{W_{1}}{\sqrt{3}}-\frac{W_{2}}{\sqrt{3}} \\
c=-\left(3+\frac{9}{4} \mu\right) W_{1}-\left(\frac{21}{4}-\frac{9 \mu}{4}\right) W_{2}
\end{gathered}
$$




$$
\begin{aligned}
d= & \frac{27}{4} \mu(1-\mu)+\frac{3}{2} \mu(1-\mu) \beta_{1}+\frac{3}{2} \mu(1-\mu) \beta_{2}+\frac{117 \mu}{4}(1-\mu) A_{1} \\
& +\frac{117 \mu}{4}(1-\mu) A_{2}-\frac{W_{1}(54-81 \mu)}{4 \sqrt{3}}-\frac{W_{2}(27-81 \mu)}{4 \sqrt{3}}
\end{aligned}
$$

The four roots of the characteristic equation of the classical RTBP is

$$
\lambda_{n}= \pm z i \quad(n=1,2,3,4)
$$

where

$$
z^{2}=\frac{1}{2}\left\{1 \pm[1-27 \mu(1-\mu)]^{\frac{1}{2}}\right\}
$$

We assume, due to oblateness and P-R drag, a solution for the equation (31) as

$$
\lambda=\lambda_{n}\left(1+\alpha_{1}+i \alpha_{2}\right)
$$

where $\alpha_{1}, \alpha_{2}$ are small real quantities.

Using (31) and neglecting second and higher order terms of small quantities we have

$$
\begin{array}{ll}
\lambda= \pm\left[-\alpha_{2}+\left(1+\alpha_{1}\right) i\right] z & \lambda^{2}=\left[-\left(1+2 \alpha_{1}\right)-2 \alpha_{2} i\right] z^{2} \\
\lambda^{3}= \pm\left\{3 \alpha_{2}-\left(1+3 \alpha_{1}\right) i\right\} z^{3} & \lambda^{4}=\left[\left(1+4 \alpha_{1}\right)+4 \alpha_{2} i\right] z^{4}
\end{array}
$$

Putting these in (22) and considering only first order terms of small quantities and since $b=b_{o}+b_{1}$, $a, b_{1}$, contain only components of small quantities, we get

$$
\begin{aligned}
& {\left[\left(1+4 \alpha_{1}\right) z^{4}-b_{o}\left(1+2 \alpha_{1}\right) z^{2}+d\right]=0} \\
& 4 \alpha_{2} z^{4} \pm a z^{3}+2 \alpha_{2} b_{o} z^{2} \pm c z=0
\end{aligned}
$$

from which we have

$$
\alpha_{1}=-\frac{\left(z^{4}-b_{o} z^{2}+d\right)}{4 z^{4}-2 z^{2} b_{o}}
$$

and

$$
\alpha_{2}=\frac{ \pm a z^{3} \mp c z}{4 z^{4}-2 b_{o} z^{2}}
$$

where the values of $a, b_{o}, c, d$ and $z$ are given in (27)-(29) and (31) respectively.

The motion around the triangular libration points is asymptotically stable only if $\alpha_{2} \neq 0$ and when the real parts, $\operatorname{Re}(\lambda)$ of the root, are all negative.

Now, $\operatorname{Re}(\lambda)=\alpha_{2} z=\frac{\mp c \pm a z^{2}}{2\left(2 z^{2}-b_{o}\right)}$ from (31), considering only the first order term $z^{2}=$ $\frac{1}{2}\left\{1 \pm\left[1-\frac{27 \mu}{2}(1-\mu)\right]\right\}$ and so taking positive sign and $\operatorname{Re}(\lambda)<0$ then,

$$
\operatorname{Re}(\lambda)=\left\{c-a\left[1-\frac{27 \mu(1-\mu)}{4}\right]\right\}\left\{2-\frac{27 \mu(1-\mu)}{2}-b_{o}\right\}^{-1}<0
$$

This implies $c\left[1+\frac{27}{2} \mu(1-\mu)\right]-a\left[1-\frac{27}{4} \mu(1-\mu)+\frac{27}{2} \mu(1-\mu)\right]<0$ and gives

$$
c+\frac{27}{4} \mu(1-\mu)(2 c-a)<a
$$

On the other hand, taking negative sign

$$
\operatorname{Re}(\lambda)=\left[-c+\frac{27 a}{4} \mu(1-\mu)\right]\left[1-\frac{27}{2} \mu(1-\mu)\right]^{-1}<0
$$


which implies $-\left[c-\frac{a 27}{4} \mu(1-\mu)\right]\left[1+\frac{27}{2} \mu(1-\mu)\right]<0$,

we have

$$
0<c+\frac{27}{4} \mu(1-\mu)(2 c-a)
$$

From eqn (36) and (37), we have

$$
0<c+\frac{27}{4} \mu(1-\mu)(2 c-a)<a
$$

as $\mu \rightarrow 0$

$$
0<c<a \quad[22]
$$

This inequality is the necessary condition for stability of triangular libration points at $L_{4}$ and $L_{5}$ according to [22]. But according to equation (29) as $\mu \rightarrow 0$

$$
c=-\left(3 W_{1}+\frac{21}{4} W_{2}\right)<0 \quad\left(W_{1}, W_{2} \geq 0\right)
$$

This contradicts the condition for stability in (38). Hence, we conclude that due to oblateness, radiation pressure, with PR-drag effect from both primaries the motion remains unstable in the linear sense.

\section{Discussion and Conclusion}

In this research, we study the effects of Poynting-Robertson drag on the location and stability of restricted three-body problem when the primaries are oblate spheroid. A system of second order non linear differential equations of motion of the system was obtained and using linear approximations we located the triangular libration points of the problem as equations (19) and (20).

We also validated the location for other generalizations by suppressing certain parameters. That is by setting any of $\beta_{1}, \beta_{2}, A_{1}, A_{2}, W_{1}, W_{2}$ to be equal to zero for the absence of radiation pressure force, oblateness and P-R drag effect respectively. The results agree with those of other researchers. The roots of the characteristics equation (22) corresponding to the variational equation (21) are given by equations (33), (34) and (35). They are found to be affected by the perturbing parameters. Due to the nature of the characteristics equation (22) we employed Murray's criteria [22] to determine the nature of its roots. We found that the triangular libration points are unstable due to the presence of radiation pressure forces, oblateness and Poynting-Robertson drag.

In particular, when $\beta_{1}=\beta_{2}=0, A_{1}=A_{2}=0$ and $W_{1}=W_{2}=0$, then $a=0, b=b_{o}=1$ and $d=\frac{27}{4} \mu(1-\mu)$, the roots of (31) gives $\lambda^{2}=\frac{-1 \pm \sqrt{1-27 \mu(1-\mu)}}{2}$ [37] and for stable motion $1>27 \mu(1-\mu)$ which implies $\mu<0.0385$. This gives the classical case.

When $A_{1}=0, A_{2}=0, \quad W_{1}=W_{2}=0$ then $a=0, b=1, c=0 \quad$ and $\quad d=\frac{3}{2} \mu(1-\mu)\left[\frac{9}{2}+\beta_{1}+\beta_{2}\right][27]$. This is the case of photo gravitational RTBP.

Also when $\beta_{1}=0, A_{1}=A_{2}=0 \quad$ and $\quad W_{2}=0 \quad$ then $\quad a=3 W_{2}, b=1+\frac{W_{1}}{\sqrt{3}}, c=-\left(3+\frac{9}{4} \mu\right) W_{1} \quad$ and $d=\frac{27 \mu(1-\mu)}{4}+\frac{3}{2} \mu(1-\mu) \beta_{1}-\frac{W_{1}(54-81 \mu)}{4 \sqrt{3}}$. This agrees with the result of [27].

When $A_{1} \neq 0, \beta_{2}=0, A_{1}=0, A_{2} \neq 0, W_{1} \neq 0$ and $W_{2}=0$. Then $a=3 W_{1}, \quad b=1+\left(\frac{3}{2}-3 \mu\right) A_{2}+\frac{W_{1}}{\sqrt{3}}$, $c=-\left(3+\frac{9}{4} \mu\right) W_{1}$ and $d=\frac{27}{4} \mu(1-\mu)+\frac{3}{2} \mu(1-\mu) \beta_{1}+\frac{117}{4} \mu(1-\mu) A_{2}-\frac{W_{1}(54-81 \mu)}{4 \sqrt{3}}$ [9]. 
When $\beta_{1}=0, \beta_{2} \neq 0, A_{1} \neq 0, A_{2}=0, W_{1}=0$ and $W_{2} \neq 0$, then, $a=3 W_{2}, \quad b=1-\left(\frac{3}{2}-3 \mu\right) A_{1}-\frac{W_{2}}{\sqrt{3}}$,

$c=-\left(\frac{21}{4}-\frac{9 \mu}{4}\right) W_{2}$ and $d=\frac{27}{4} \mu(1-\mu)+\frac{3}{2} \mu(1-\mu) \beta_{2}+\frac{117}{4} \mu(1-\mu) A_{1}-\frac{W_{2}(27-81 \mu)}{4 \sqrt{3}}$ [34].

The roots of the characteristics equation (22) corresponding to the variational equation (30) is given by the equations (33), (34) and (35) where values of $a, b, c$ are given in (27) to (30). These equations all depend on the parameters of the perturbing factors.

The nature of the value of $c$ as compared to the condition necessary for stability by Murray [22] shows that due to radiation pressure, forces, oblateness and Poynting-Robertson drag from primaries the motion of the infinitesimal body remains unstable.

Successive research results of generalizations involving radiation pressure forces, oblateness of primaries and Poynting-Robertson drag have shown that they are destabilizing forces. Astrophysical evidences have also revealed that these parameters are natural activities in our solar, extrasolar and stellar systems. A satellite (natural or artificial) is expected to navigate in the neighbourhood of the planets in our solar system in their stable orbits under the influence of these forces. Hence, our result provides information for a space/astronomical engineers to take into consideration, the destabilizing effects of radiation pressure forces, oblateness of the primaries and Poynting-Robertson drag when designing a spacecraft that will navigate in the vicinity of the planets.

\section{References}

1. E.I. Abouelmag, "Existence and Stability of Triangular Point in the Restricted Three-Body Problem with Numerical Applications," Astrophysics and Space Science, vol. 342, pp. 48-53, 2012.

2. S.B. Akere-Jaiyeola, J. Singh, A, Abdulraheem and J. Braimah, "On the Effects of Perturbation, Radiation and Triaxiality on the Stability of Libration Points in the Restricted Three-body Problem," IOSR journal of mathematics, vol. 11, no. 1, pp. 69-79, 2015.

3. A. Abdulraheem, and J. Singh," Combined Effects of Perturbations Radiations and Oblateness on the Stability of Equilibrium Points in the Restricted Three-body Problem," Astronomical Journal, vol. 131, pp. 1880-1885, 2006.

4. J. A. Aredondo, J. Gui and C. Stoica, "On the Restricted Three-body Problem with Oblate Primaries," Astrophysics and Space Sci, vol. 341, pp. 315 -322, 2012.

5. K. B. Bhatnagar and P. P. Hallan, "Effects and Perturbations in the Coriolis and Centrifugal Forces on the Stability of Libration Points in the Restricted Problem," Cellestial Mechanics, vol. 18, pp. 105-112. 1979).

6. K.B. Bhatnagar and M.L. Khanna, "Existence and Stability of Libration Points in the Restricted Three-Body Problem when the small Primary is triaxle Rigid Body and the Bigger one is an Oblate Spheroid," Indian Journal of Pure and Applied Mathematics, vol. 30, no. 7, pp. 721-733, 1999.

7. Y.A. Chernikov." The Photogravitational Restricted Three-body Problem," Soviety Astronomy Astrophysics, vol. 14, no. 1, pp. $176-181,1970$.

8. M.K. Das, P. Narang, S. Mahajan and M. Yuasa, "On Out of Plane Equilibrium Points in Photogravitational Restricted Three-Body Problem," Journal of Astronomical. Astrophysics, vol. 30, pp. 177-185. 2009.

9. B. Ishwar and B.S. Kushvah," Linear Stability of Triangular Equilibrium Points in the Generalized Photogravitational Restricted Three-Body Problem with Poynting-Robertson drag," Journal of Dynamical system, vol. 4, no.1, pp. 79-86, 2006.

10. B. Ishwar and J. Singh," Stability of Triangular Points in Generalized Photogravitational Restricted ThreeBody Problem," Bulletin of Astronomical Society of India, vol.27, pp. 415 -424.1999.

11. M. Jain, S.B. Chakraborty and Abdullah," Restricted Three-body Problem with Robertson Drag Effect," International Journal of Applied Mathematics and Mechanics, vol. 10, no. 3, pp. 32-44, 2013.

12. S. N.Khasan,"Libration Solutions to the Photogravitaional Three-Body Problem," Cosmic Research, vol. 34, no. 2, pp.146 -151, 1996. 
13. V. Kumar and R. K. Choudhry, "On the Stability of the Triangular Libration Points for the Photogravitaional Circular Rescrited Problem of Three Bodies when both of the Attracting Bodies are well Radiating," Cellestial Mechanics, vol. 40, pp. 155-170, 1987.

14. A. L. Kunitsyn and E. N. Polyathara, "The Restricted Photogravitational Three-body problem," Astronomical Astrophysics Trans, vol. 6, pp. 283-293, 1995.

15. A. L. Kunitsyn and A. A. Perezhogin, "On the Stability of Triangular Libration Points of the Photogravitational Restricted Circular Three-body Problem," Celestial Mechanics, vol. 18, pp. 395-408, 1978.

16. A. L. Kunitsyn, "Stability of Triangular Libration Point in the Photogravitational Three-body Problem," Journal of Applied Mathematics and Mechanics, vol. 65, no. 5, pp. 757-760. 2000.

17. A. L. Kunitsyn, "Stability of Colinear Libration Point in the photogravitional Three-Body Problem," Journal of Applied Mathematics and Mechanics, vol. 65 no. 4, pp. 703-706, 2001.

18. B. S. Kushvah and B. Ishwar, "Triangular Equilibrium Points in the Generalized Photogravitational Restricted Three-Body Problem with Poynting-Robertson Drag,". Review Bull. Cal. Math. Soc, vol. 12, no. 1 2, pp. 109-114, 2004.

19. D. C. Liou, H. A. Zook and A.A. Jackson, "Radiation Pressure Poynting Robertson drag and Solar wind drag in the Restricted Three-body" Icarus, vol. 116, pp. 186-201, 1995.

20. S. W. Mc Cuskey, Introduction to Celestial Mechanics, 1963.

21. F. Mignard, "Stability of L_4 and L_5 Against Radiation Pressure," Celestial Mechanics, vol. 34, pp. 275-287, 1984.

22. C. D. Murray," Dynamical Effect of Drag in the Circular Restricted Three-Body Problem Location and Stability of Lagrangian Equilibrium Points," Icarus, vol. 112, pp. 465-484, 1994.

23. J. H. Poynting," Radiation in the Solar System: Its effect on Temperature and its Pressure on small Bodies,". MNRAS, vol. 64, A1, 1903.

24. Radzievskii, V.V, "The Restricted Problem of Three-Bodies Taking Account of Light Pressure," Akad. Nauk. USSR Astronomical Journal, vol. 2, pp. 250-256, 1950.

25. O, Ragosand F. A. Zafiropoulos, "A Numerical Study of the Influence of the Poynting Robertson Effect on the Equilibrium Points of the Photogravitational Restricted Three-body problem Coplanar case," Astronomical Astrophysics, vol. 300, pp. 568-578, 1995.

26. H. P. Robertson, "Dynamical Effects of Radiation in the Solar System," MNRAS, vol. 97,423.

27. D. W. Schuerman, "The Restricted Three-Body Problem Including Radiation Pressure," Astrophysics Journal, vol. 238, no. 1, pp. 337-342, 1980.

28. P. Sengupta and P.Singla, "An Analysis of Stability in the Restricted Three-Body Problem," MEEN 689 project, 2002.

29. S.M. Shaboury, "Equilibrium Solution of the Restricted Problem of $2+2$ Axisymmetric Rigid-Bodies,"Celestial Mechanics and Dynamics Astronomy,vol. 50, pp. 199-208, 1991.

30. R. K.Sharma and P. V. Subbarao, "Stationary Solutions and Their Characteristics Exponents in the Restricted Three-body Problem when the More Massive Primary is an Oblate Spheroid," Cellestial Mechanics, vol. 13, pp. 137-149, 1976.

31. R. K. Sharma, Z. A. Taqvi and K. B. Bhatnagar. "Existence and Stability of Libration Points in the Restricted Three-body Problem when the Primaries are Triaxial Rigid bodies,".Cellestial Mechanics and Dynamical Astrophysics ,vol. 79, pp. 119-133, 2001a.

32. R. K. Sharma, Z. A. Taqvi and K. B. Bhatnagar, "Existence and Stability of Libration Points in the Restricted Three-body Problem when the Primaries are Triaxial Rigid bodies and Source of Radiations," Indian Journal of Pure Applied Mathematics,vol. 32, no. 7, pp. 981-994.2001b.

33. J.F.L. Simmons, A.J.C. Mc Donald,, Brown, J. C. (1985). The Restricted 3-Body Problem with Radiation Pressure.Celestial Mechanics, 35(2), 145-187.

34. J. Singh and T. O. Amuda, "Poynting-Roberson Drag and Oblateness Effects on Motion Around the Equilibrium Points in the Photogravitational R3BP, "Astrophysics and Space Science,vol. 350, pp. 119-126, 2014.

35. J. Singh, J. Taura, J. Joel, "Stability of Equilibrium Points in a Circular Restricted Three-body Problem with Oblate Bodies Enclosed by a Circular Cluster of Material Points," Astrophysics and Space Science,vol. 349, pp. 681-697, 2014. 
36. J. Singh, "Combined Effect of Perturbations, Radiation and Oblateness on the Nonlinear Stability of Triangular Point in the restricted Three-Body Problem," .Astrophysics and Space science,vol. 332,no. 2, pp. 331-339,2011.

37. V. Szebehely, Theory of Orbits. Academic Press, 1967.

38. V. Szebehely, "Stability of the Point of Equilibrium in the Restricted Problem," Astronomical Journal, vol. 72, no. 1, pp. 7-9, 1967a.

39. V. V. Vidyakin, "A Plane Circular Limited Task Pertaining to Three Spheroids," Astronomical Journal, vol. 51, no. 5, pp. 1087-1094, 1974 